\title{
Acolhimento de Enfermagem na Unidade de Terapia Intensiva (UTI) neonatal segundo binômio pais-filhos: estudo de revisão integrativa da literatura
}

\author{
Nursing care at the neonatal intensive care unit (ICU) according to parent-child binomial: \\ integrative literature review study
}
Atención de enfermería en la unidad de cuidados intensivos neonatales (UCI) según el binomio padre-hijo: estudio de revisión de literatura integradora

Deisiane da Silva Mesquita ${ }^{1 *}$, Karytta Sousa Naka ${ }^{1}$, Ana Paula Sardinha Kawamura², Andreia Sabrina Schmidt².

\section{RESUMO}

Objetivo: analisar o conhecimento exposto na literatura sobre o acolhimento de enfermagem na UTI neonatal (NEO) segundo o binômio mãe-filho. Métodos: revisão integrativa da literatura, com consulta nas bases de dados LILACS, PUBMED e SCIELO. Utilizou-se os descritores: UTI neonatal AND acolhimento, UTI neonatal AND humanização, UTI neonatal AND relação pais-bebês. Foram selecionados 14 artigos de acordo com os critérios de inclusão, entre os anos de 2011 a 2018. Resultados: Foram incluídos 14 artigos na amostra final do estudo, estando categorizados em: Assistência de enfermagem em UTI neonatal; Percepção dos pais sobre seu filho internado na UTI NEO; Vínculo entre pais-filhos em UTI neo; Vivência dos pais na UTI NEO Humanização na UTI NEO sob a ótica dos pais. Conclusão: O papel da enfermagem é intenso com grandes responsabilidades com o recém-nascido e com o acolhimento dos pais, prestando orientações adequadas sobre os cuidados e realizando intervenções fortalecendo o enfrentamento de medos, angústias e dúvidas.

Palavras-Chave: Enfermagem Neonatal; Assistência Integral à saúde; Unidades de Terapia Intensiva Neonatal; Cuidados de Enfermagem; Saúde da Criança.

\begin{abstract}
Objective: to analyze the knowledge in the literature about the nursing reception at neonatal ICU according to the parents-children binomial. Methods: This is a integrative review of the literature, with consultation in the LILACS, PUBMED and SCIELO databases. The descriptors: neonatal ICU and host, neonatal ICU and humanization, neonatal ICU and parents-infant relationship. Fourteen articles were selected according to the inclusion criteria, between the years 2011 to 2018. Results: Fourteen articles were included in the final sample of the study, being categorized into: Nursing care in neonatal ICU; Perception of the parents about their child hospitalized in the neonatal ICU; Parent-child link in neo-ICU; Parents' experience in the neonatal ICU; Humanization in the Neonatal ICU from the perspective of the parents. Conclusion: The role of the child is of great importance to the newborn and to the reception of the parents, and the persuasions are precarious and conscious.
\end{abstract}

Keywords: Neonatal Nursing; Comprehensive health care; Neonatal Intensive Care Units; Nursing care; Child Health.

\footnotetext{
1 Instituto Evandro Chagas (IEC/SVS/MS), Ananindeua-Pará. *E-mail: deisi_mesquita@hotmail.com

2 Faculdade Conhecimento e Ciência (FCC), Belém-Pará.
} 


\section{RESUMEN}

Objetivo: analizar el conocimiento en la literatura sobre la acogida de enfermería en la UTI neonatal según el binomio madre-hijos. Métodos: estudio descriptivo del tipo de revisión integrativa de la literatura, con consulta en las bases de datos LILACS, PUBMED y SCIELO. Se utilizó los descriptores: UTI neonatal y acogida, UTI neonatal y humanización, UTI neonatal y relación padres-bebés. Se seleccionaron 14 artículos de acuerdo con los criterios de inclusión, entre los años de 2011 a 2018. Resultados: Se incluyeron 14 artículos en la muestra final del estudio, estando categorizados en: Asistencia de enfermería en UTI neonatal; Percepción de los padres sobre su hijo internado en la UTI neo; Vínculo entre padres-hijos en UTI neo; Vivencia de los padres en la UTI neo; Humanización en la UTI neo bajo la óptica de los padres. Conclusión: El papel del niño es de gran importancia con el recién nacido y con la acogida de los padres, y las persuasiones son precarias y conscientes.

Palabras clave: Enfermería Neonatal; Asistencia integral a la salud; Unidades de Terapia Intensiva Neonatal; Cuidados de Enfermería; Salud del Niño.

\section{INTRODUÇÃO}

O nascimento é considerado um fenômeno de vulnerabilidade, pois o recém-nascido atravessa uma série de transformações no momento da passagem da vida intrauterina para o extra útero o nascimento, fazendo com que esse período definido como neonatal (que compreende do nascimento até 28 dias de vida), seja considerado crítico. Durante esse processo de transição, podem ocorrem intercorrências ou alterações fisiológicas que implicam no desenvolvimento não sadio (NETO JAS e RODRIGUES BMR, 2010; SOUZA ABG, 2014).

Em meio a esse processo, ocorrem algumas intercorrências ou podem ser observadas algumas alterações fisiológicas que retardaram o desenvolvimento normal e saudável da criança. Nesses momentos se faz necessário a utilização da unidade de terapia intensiva neonatal (UTIN) (COELHO AS, et al., 2018).

A UTIN está destinada ao atendimento de neonatos com até 28 dias de vida, que necessitem de um suporte assistencial intensivo. Com apoio de suporte vital completo, monitoração, equipamentos de reanimação, entre diversos outros e serviço auxiliar de apoio. Que fornecem condições essenciais e vitais para a sobrevivência e desenvolvimento do $R N$ no ambiente extrauterino (SOUZA ABG, 2014).

Este serviço possui diretrizes e objetivos definidos na Portaria no 930, de 10 de maio de 2012, divulgada pelo Ministério da Saúde (MS), a qual estabelece a atenção integral e humanizada ao recémnascido, incluindo: "o respeito, a proteção e o apoio aos direitos humanos; atenção humanizada; estímulo à participação e ao protagonismo da mãe e do pai nos cuidados ao recém-nascido" (BRASIL, 2011).

E apesar de ser importante para assegurar a vida, é considerada um ambiente estressante e tenso, impessoal e temeroso, por ser um ambiente repleto de luzes fortes, barulho, mudanças de temperatura e interrupção do ciclo do sono por conta das repetidas avaliações e procedimentos, acarretando, muitas vezes, em desconforto e dor (SOUZA ABG, 2014).

Dados do MS afirmam que a UTIN é um ambiente onde podem ocorrer inúmeras intervenções, fazendo com que o bebê seja manipulado excessivamente pelos profissionais de saúde, podendo chegar ao número de 488 procedimentos/dia, causando o surgimento de fatores estressantes para o recémnascido (BRASIL, 2011).

Neste contexto, a equipe de enfermagem se encontra exposta a uma complexidade psicológica, física e emocional devido a presença da família que passa por um momento de insegurança em relação a vida do bebê. Na literatura vários autores destacam o papel da enfermagem nas UTIN, desde como facilitadora ou provedora de um acolhimento humanizado, quanto à assistência em saúde com 
competência e habilidade teoria-prática de acordo com os protocolos preconizados (SEGANTINI FLC, et al., 2018; COSTA R e PADILHA MI, 2011). Um dos papéis da enfermagem consiste na diminuição dos estressores da UTIN e na implantação de ações que resultem em um ambiente terapêutico, que seja agradável e acolhedor tanto ao neonato como também aos seus familiares. Por passar mais tempo na assistência, a equipe de enfermagem reconhece melhor os sinais de desconforto dos recémnascidos, podendo atuar de forma eficaz e humanizada (FERREIRA CM, 2011).

No que se refere ao acolhimento humanizado nesse ambiente crítico, a assistência deverá ser sensível ao binômio mãe-filho, de forma que satisfaça as necessidades independentes do prognóstico da criança, pois a assistência deve ser promovida de forma a transmitir o devido respeito em relação à dor dos familiares, principalmente dos pais (MENON D, et al., 2008).

Diante do exposto o presente trabalho tem o objetivo analisar o conhecimento exposto na literatura sobre o acolhimento de enfermagem na UTI neonatal segundo o binômio pais-filhos.

\section{MÉTODOS}

Trata-se de uma revisão integrativa da literatura, baseado nas seguintes etapas: 1) estabelecer a hipótese ou questão de pesquisa; 2) amostragem ou busca na literatura; 3) categorização dos estudos; 4) avaliação dos estudos; 5) interpretação dos resultados e, por fim, 6) síntese do conhecimento ou apresentação da revisão (MENDES KDS, et al., 2008).

Foram utilizadas três bases de dados: Literatura Latino-Americana e do Caribe em Ciências da Saúde (LILACS), Banco de Dados de Trabalhos na Área da Saúde (PUBMED) e Scientific Eletronic Library Online (SCIELO).

A busca foi norteada com a associação dos descritores pesquisados nos idiomas português e inglês: "UTI neonatal AND acolhimento"/“UTI neonatal AND host”; "UTI neonatal AND humanização"/“UTI neonatal AND humanization" e "UTI neonatal AND relação pais-bebês"/“UTI neonatal AND baby fathers relationship".

Os critérios de inclusão foram estudos realizados entre os anos de 2011 a 2018; textos completos disponíveis online; idiomas inglês, espanhol ou português e que abordassem sobre acolhimento em UTI neonatal. Não foram incluídos estudos repetidos.

Após a análise dos estudos, seguindo os critérios de inclusão e exclusão, foram encontrados 1.002 artigos. A primeira etapa de seleção obteve 85 artigos com base na leitura do título.

Em seguida foi realizada a leitura dos resumos dos artigos selecionados, separando-se 44 artigos. Ao final do processo, foram analisados integralmente 22 artigos e, após a exclusão de duplicidade, a amostra final contou com 14 artigos.

A construção da análise de dados foi baseada na técnica proposta por Stillwell, et al. (2010) sobre nível de evidência no qual agrupa os trabalhos da seguinte forma: I- revisão sistemática ou metanálise; II- Ensaio Clínico Randomizado Controlado; III- Ensaio Clínico Sem controlado; IV- caso controle ou estudo coorte; V- Revisão sistemática de estudo qualitativo ou descritivo; VI- Estudo qualitativo ou descritivo; VII - Artigo de opinião ou consenso de órgãos governamentais ou conselho de especialidades médicas.

Assim, os dados obtidos foram dispostos em quadros que incluem as informações do nível de evidência e categorização dos estudos.

\section{RESULTADOS}

Com referência ao ano de publicação, notou-se que dos 14 artigos selecionados, o ano de 2013 apresentou $43,0 \%$ das publicações, enquanto que o ano de 2016 não apresentou nenhuma (Quadro 1). 
Quadro 1 - Distribuição dos artigos conforme o ano de publicação.

\begin{tabular}{|l|l|l|}
\hline ANO DE PUBLICAÇÃO & N & $\%$ \\
\hline 2011 & 2 & 15,0 \\
\hline 2012 & 1 & 7,0 \\
\hline 2013 & 6 & 43,0 \\
\hline 2015 & 1 & 7,0 \\
\hline 2016 & 1 & 7,0 \\
\hline 2017 & 0 & 0,0 \\
\hline 2018 & 1 & 7,0 \\
\hline TOTAL & 2 & 14,0 \\
\hline
\end{tabular}

Fonte: Mesquita DS et al. (2019).

Com base no nível de evidência dos estudos selecionados, observou-se que a maioria dos estudos apresentou delineamento de revisão sistemática de estudo qualitativo ou descritivo (Quadro 2).

Quadro 2 - Delineamento dos estudos selecionados na amostra final segundo o nível de evidência.

\begin{tabular}{|l|c|c|}
\hline \multicolumn{1}{|c|}{ DELINEAMENTO } & NÍVEL DE EVIDÊNCIA & N \\
\hline Revisão sistemática ou metanálise & I & 2 \\
\hline Ensaio Clínico Randomizado Controlado & III & 0 \\
\hline Ensaio Clínico Não Randomizado & IV & 0 \\
\hline Caso controle ou estudo coorte & V & 11 \\
\hline Revisão sistemática de estudo qualitativo ou descritivo & VI & 0 \\
\hline Estudo qualitativo ou descritivo & VII & 1 \\
\hline Estudo quantitativo & & 14 \\
\hline Total & & \\
\hline
\end{tabular}

Fonte: Mesquita DS et al., (2019).

A leitura e a interpretação amostral dos trabalhos permitiram analisá-los de acordo as seguintes categorias: Categoria A: Assistência de enfermagem em UTIN; Categoria B: Percepção dos pais sobre seu filho internado na UTIN; Categoria C: Vínculo entre pais-filhos em UTIN; Categoria D: Vivência dos pais na UTIN; Categoria E: Humanização na UTIN sob a ótica dos pais (Quadro 3). 
Quadro 3 - Síntese dos estudos incluídos no estudo. Belém - PA, 2019.

\begin{tabular}{|c|c|c|c|}
\hline AUTORES - ANO & TÍTULO & CATEGORIAS & $\begin{array}{l}\text { PRINCIPAIS RESULTADOS } \\
\end{array}$ \\
\hline $\begin{array}{l}\text { FRELLO AT, CARARO TE., } \\
2012\end{array}$ & $\begin{array}{l}\text { Enfermagem e a relação com as } \\
\text { mães de neonatos em unidade de } \\
\text { terapia intensiva neonatal. }\end{array}$ & $\begin{array}{l}\mathrm{A} \\
\mathrm{B}\end{array}$ & $\begin{array}{l}\text { A necessidade das mães por suporte inclui informações } \\
\text { detalhadas e em linguagem acessível acerca do estado clínico e } \\
\text { procedimentos realizados com o filho, apoio emocional, } \\
\text { envolvimento nos cuidados ao bebê, disponibilidade para estar e } \\
\text { conversar com as mães. }\end{array}$ \\
\hline $\begin{array}{l}\text { TURNER M, WINEFIELD H, } \\
\text { CHUR-HANSEN A., } 2013\end{array}$ & $\begin{array}{l}\text { The emotional experiences and } \\
\text { supports for parents with babies in a } \\
\text { neonatal nursery. }\end{array}$ & $\begin{array}{l}\text { C } \\
\text { D }\end{array}$ & $\begin{array}{l}\text { O grupo de apoio foi relatado para ser eficaz e útil para os pais. } \\
\text { Os pais geralmente mantinham contato social com outros } \\
\text { pais e recorriam a conselhos e informações de apoio do grupo. }\end{array}$ \\
\hline SOARES RLSF, 2013 & $\begin{array}{l}\text { O significado de ter um filho } \\
\text { prematuro para pais homens: } \\
\text { contribuições da enfermagem } \\
\text { neonatal. }\end{array}$ & $\begin{array}{l}\text { A } \\
B\end{array}$ & $\begin{array}{l}\text { É preciso aprender a trabalhar com a nova realidade cultural onde } \\
\text { os homens cuidam do lar e dos filhos junto com as mulheres. É } \\
\text { urgente a conscientização dos profissionais para entenderem o } \\
\text { movimento cultural atual que envolve o papel do homem enquanto } \\
\text { pai e a necessidade de apoio à paternidade nas situações de } \\
\text { prematuridade. }\end{array}$ \\
\hline $\begin{array}{l}\text { SOARES LG, et al., } \\
2014\end{array}$ & $\begin{array}{l}\text { Enfermagem neonatal em cuidados } \\
\text { intensivos: o olhar das famílias. }\end{array}$ & $\begin{array}{l}\text { A } \\
B \\
E\end{array}$ & $\begin{array}{l}\text { Apesar do pouco contato dos pais com a Enfermagem no local de } \\
\text { estudo, foi possível concluir que estes reconhecem a figura do } \\
\text { enfermeiro, enfatizam a humanização do cuidado, porém não } \\
\text { percebem competências gerenciais e utilização de conhecimento } \\
\text { científico na prática do enfermeiro. }\end{array}$ \\
\hline $\begin{array}{l}\text { MEDEIROS } \\
\text { PICCININI CA., } 2015\end{array}$ & $\begin{array}{l}\text { Relação pai-bebê no contexto da } \\
\text { prematuridade: } \quad \text { gestação, } \\
\text { internação do bebê e terceiros mês } \\
\text { após a alta hospitalar. }\end{array}$ & $\begin{array}{l}\text { C } \\
D\end{array}$ & $\begin{array}{l}\text { A importância das visitas frequentes ao bebê durante a internação, } \\
\text { para o estabelecimento da relação. Ficou evidente o receio de } \\
\text { contato físico, em especial nas primeiras semanas de } \\
\text { hospitalização, o que deve ser considerado nas intervenções pai- } \\
\text { bebê durante a internação. }\end{array}$ \\
\hline LIMA VF, et al., 2017 & $\begin{array}{l}\text { Vivência dos familiares de } \\
\text { prematuros internados em unidade } \\
\text { de terapia intensiva neonatal. }\end{array}$ & $\begin{array}{l}\text { C } \\
\text { D }\end{array}$ & $\begin{array}{l}\text { É necessário mudar a filosofia dos profissionais e das instituições, } \\
\text { na perspectiva do cuidado à família, para que este possa } \\
\text { ultrapassar a dimensão biológica, com espaços de diálogo, para } \\
\text { que a família faça parte do cuidado. }\end{array}$ \\
\hline $\begin{array}{l}\text { SIQUEIRA MBC, DIAS } \\
\text { MAB., } 2011\end{array}$ & $\begin{array}{l}\text { A percepção materna sobre } \\
\text { vivencia e aprendizado de cuidado } \\
\text { de um bebê prematuro. }\end{array}$ & $\begin{array}{l}\text { B } \\
D\end{array}$ & $\begin{array}{l}\text { Necessidade de maior organização da assistência materno- } \\
\text { infantil, com incorporação da integralidade do cuidado e } \\
\text { humanização da relação entre a família e os profissionais de } \\
\text { saúde. }\end{array}$ \\
\hline
\end{tabular}




\begin{tabular}{|c|c|c|c|}
\hline $\begin{array}{l}\text { SILVA PLN, et al., } \\
2018\end{array}$ & $\begin{array}{l}\text { Vivência e necessidade de pais de } \\
\text { neonatos prematuros internados } \\
\text { em unidade de terapia intensiva } \\
\text { neonatal }\end{array}$ & $\begin{array}{l}B \\
D\end{array}$ & $\begin{array}{l}\text { Necessidade de maior organização da assistência materno- } \\
\text { infantil, com incorporação da integralidade do cuidado e } \\
\text { humanização da relação entre a família e os profissionais de } \\
\text { saúde. }\end{array}$ \\
\hline NODA LN, et al., 2018 & $\begin{array}{l}\text { A humanização em unidade de } \\
\text { terapia intensiva neonatal sob a } \\
\text { ótica dos pais }\end{array}$ & $\begin{array}{l}\text { A } \\
D \\
E\end{array}$ & $\begin{array}{l}\text { O cuidado humanizado emerge como a maneira que se cuida e } \\
\text { pela relação com os profissionais de saúde. }\end{array}$ \\
\hline $\begin{array}{l}\text { SANTANA } \\
\text { MADM, } \\
\text { MADEIRA LM., } 2013\end{array}$ & $\begin{array}{l}\text { A mãe acompanhante na unidade } \\
\text { de terapia intensiva desafios para } \\
\text { equipe assistencial }\end{array}$ & $\begin{array}{l}\text { A } \\
E\end{array}$ & $\begin{array}{l}\text { A comunicação efetiva entre as mães e a equipe assistencial foi } \\
\text { apontada como principal estratégia a ser utilizada para } \\
\text { minimização dos conflitos. O trabalho multiprofissional é } \\
\text { ressaltado, além do reconhecimento dos aspectos emocionais } \\
\text { das mães e a interação com a família, a fim de proporcionar } \\
\text { segurança, afetividade e atendimento qualificado. }\end{array}$ \\
\hline $\begin{array}{l}\text { NASCIMENTO CAD, } \\
\text { et al., } 2013\end{array}$ & $\begin{array}{l}\text { Percepção dos enfermeiros sobre } \\
\text { os pais de prematuros em UTI } \\
\text { neonatal }\end{array}$ & $\begin{array}{l}\text { A } \\
\text { B } \\
\text { C } \\
\text { D }\end{array}$ & $\begin{array}{l}\text { Os pais, em sua relação com filho prematuro e interno na UTIN, } \\
\text { apresentam-se, segundo os enfermeiros, com uma condição } \\
\text { emocional de angústia caracterizada pela ruptura entre a imagem } \\
\text { previamente formada e desejada, a de uma criança saudável, e } \\
\text { aquela oriunda da prematuridade: uma criança adoecida e com } \\
\text { reais possibilidades de sequelamento ou óbito }\end{array}$ \\
\hline $\begin{array}{l}\text { OLIVEIRA K, et al., } \\
2013\end{array}$ & $\begin{array}{l}\text { Vivência familiar no processo de } \\
\text { nascimento e internação de seus } \\
\text { filhos em UTI }\end{array}$ & $\begin{array}{l}\text { C } \\
\text { D }\end{array}$ & $\begin{array}{l}\text { O estudo evidenciou a importância de envolver a família no } \\
\text { processo assistencial como fator precursor da qualidade da } \\
\text { atenção humanizada. }\end{array}$ \\
\hline $\begin{array}{l}\text { RAMADA NCO, et al., } \\
2013\end{array}$ & $\begin{array}{l}\text { Toque terapêutico: influência nos } \\
\text { parâmetros vitais de recém- } \\
\text { nascidos }\end{array}$ & $\begin{array}{l}\mathrm{C} \\
\mathrm{E}\end{array}$ & $\begin{array}{l}\text { Os resultados evidenciam que o toque terapêutico promove o } \\
\text { relaxamento do recém-nascido, favorecendo a redução dos } \\
\text { parâmetros vitais e, consequentemente, a taxa de metabolismo } \\
\text { basal. }\end{array}$ \\
\hline SPIR EG, et al., 2011 & $\begin{array}{l}\text { A percepção do } \\
\text { acompanhante sobre a } \\
\text { humanização da assistência em } \\
\text { uma unidade neonatal. }\end{array}$ & $\begin{array}{l}\text { A } \\
B\end{array}$ & $\begin{array}{l}\text { Constatou-se satisfação quanto à assistência recebida e ao apoio } \\
\text { da equipe, mas foram apontadas necessidades de mudanças na } \\
\text { estrutura física do setor, para proporcionar uma assistência mais } \\
\text { humanizada. }\end{array}$ \\
\hline
\end{tabular}

Fonte: Mesquita DS et al. (2019).

A humanização em unidade de sob a

A mãe acompanhante na unidade

de terapia intensiva desafios para

os pais de prematuros em UTI neonata

ma unidade neonatal.
Necessidade de maior organização da assistência maternoinfantil, com incorporação da integralidade do cuidado $e$ saúde.

O cuidado humanizado emerge como a maneira que se cuida $e$

apontada como principal estratégia a ser utilizada para minimização dos conflitos. O trabalho multiprofissional e segurança, afetividade e atendimento qualificado.

Os pais, em sua relação com filho prematuro e interno na UTIN, apresentam-se, segundo os enfermeiros, com uma condição emocional de angústia caracterizada pela ruptura entre a imagem previamente formada e desejada, a de uma criança saudável, e aquela oriunda da prematuridade: uma criança adoecida e com eais possibilidades de sequelamento ou óbito basal. humanizada.

REAS/EJCH | Vol. 11(13) | e980 | DOI: https://doi.org/10.25248/reas.e980.2019 Página 6 de 8 


\section{DISCUSSÃO}

A importância do trabalho em equipe multidisciplinar em uma UTIN destaca o papel fundamental do profissional de enfermagem como provedor do cuidado, assistência gestão e acolhimento. Apesar de o enfermeiro estar conquistando destaque na área da gestão, as maiorias dos estudos analisados associam e descrevem sobre a assistência. Em relação à assistência dentro do ambiente da UTIN, o enfermeiro requer atenção máxima para lidar com o recém-nascido, equipamentos, procedimentos mais avançados e decisões clínicas que interferem no tratamento (COELHO AS, et al., 2018).

Na UTIN, a assistência de enfermagem é intensa, pois são responsáveis tanto pelo manuseio dos equipamentos tecnológicos, pelo acolhimento dos pais na visita ao filho quanto pela orientação sobre os cuidados inerentes ao tratamento. Incluí-los no planejamento da assistência, bem como respeitar suas decisões acerca do tratamento, caracterizam um tipo de assistência orientada por escuta e intervenção necessária ao enfrentamento de medos, angústias e dúvidas (STILLWELL SB, et al., 2010).

O suporte da enfermagem na UTIN possui além das responsabilidades com o neonato, compromisso junto aos pais, em especial as mães, e muitas atividades são elencadas nos estudos como fundamentais para serem desenvolvidas junto à família durante a internação do bebê, dentre elas: acompanhá-los nas primeiras visitas, informar sobre as condições do bebê, responder as questões e fornece suporte emocional sendo empático, compreensivo e encorajando a visita o toque, envolver nos cuidados, informar acerca dos procedimentos e tratamentos realizados (COELHO AS, et al., 2018; NASCIMENTO CAD, et al., 2013; SANTOS MSN, et al., 2018).

A internação de um filho em UTIN é uma experiência difícil e desafiadora para as mães e suas famílias, uma vez que os ambientes altamente modernos e tecnológicos separam os bebês fisicamente, psicologicamente e emocionalmente de seus pais. Além disso, adicionam-se diversos problemas a serem enfrentados pelas famílias durante o período de internação, entre os quais, a vivência da separação do bebê, o medo da doença, do desconhecido, do ambiente hospitalar e a incerteza da evolução clínica do bebê e de sua sobrevivência (CAMPONOGARA S, et al., 2018).

É conhecido que durante a internação dos filhos, mães compreendem a fragilidade do filho com a necessidade de cuidados especiais, no entanto, sentem à vontade de cuidar ou ajudar no cuidado. Contudo, a ausência ou inadequada informação oferecida pelos profissionais de saúde as mães de como prover esse cuidado podem desencadear, até mesmo uma depressão pós-parto (VERONEZ M, et al., 2017; COUTO FF e PRAÇA NS, 2012).

Quando um RN necessita da internação na UTIN, os pais vivenciam a incerteza, culpa, medo da perda e insegurança. Diante da fragilidade da internação do filho, muita das vezes a mãe pode desenvolver a sensação de fracasso por não prover o cuidado ao filho, inutilidade e inferioridade, podendo ter dificuldade de se reconhecer como mãe. Isso pode comprometer o vínculo entre pais e filhos devido ao distanciamento físico e emocional (CAMPONOGARA S, et al., 2018). Na literatura, comumente é descrito o vínculo entre mãe e filho, onde não se reconhece o pai capaz de oferecer cuidados. Segundo Siqueira MBC e Dias AMB (2011) em estudo com experiências de pais com filhos internados na UTIN, os pais descreveram o medo da perda, insegurança, angústia, rotina alterada e a espera ansiosa da alta hospitalar. Assim, consideraram que o pai necessita de um olhar mais atencioso por parte da equipe assistencial, muitas vezes negligenciado.

Para Rodrigues LM e Moreira PL (2013), na unidade neonatal o cotidiano protagonizado pelos pais e equipe de saúde é permeado por conflitos, manifestado por meio de indiferença, frieza e até desrespeito mútuo. Mencionam ainda que a presença do acompanhante pode ser vista pela equipe como causa de dificuldade e até incomodo dentro da unidade. A comunicação efetiva entre os pais/familiares e a equipe assistencial como principal estratégia para a solução e redução dos conflitos, podendo ser determinante para que os pais se sintam seguros num ambiente estranho, considerando-se sua fragilidade e seus sentimentos frente à internação do recém-nascido na UTIN (SANTANA EFM e MADEIRA KM, 2013; CARTAXO LS, et al., 2014). 
Nesse sentido, a abordagem e acolhimento da família são extremamente importantes para a efetivação desta aproximação e participação no cuidado, devendo ser valoriza. Além disso, é necessária a valorização mútua, onde os familiares possam compreender as intervenções da equipe, e a mesma possa os aspectos emocionais que permeiam a situação buscando proporcionar segurança, atendimento qualificado e humanizado.

\section{CONSIDERAÇÕES FINAIS}

Por meio dos dados encontrados no presente estudo, conclui-se que a literatura aborda a importância da presença das mães na UTIN para oferecer afeto, segurança e conforto para o filho. Além disso, foi possível destacar o benefício da comunicação efetiva dos profissionais com os pais em relação ao estado clínico do recém-nascido, incluindo as informações detalhadas com uma linguagem acessível de acordo com cada cultura e os procedimentos realizados com o recém-nascido na UTIN. Ademais, a pesquisa revelou que a enfermagem deve se adaptar à nova realidade cultural onde os homens apresentam um papel diferenciado como de costume da sociedade, como por exemplo, cuidam do lar e dos filhos juntos com as mulheres. Dessa maneira, a sensibilização dos profissionais para entender essa mudança e permitir a inserção do pai com o bebê se faz necessária, garantido o apoio à paternidade nas situações de internações em UTIN. Por fim, o papel do enfermeiro está presente em todas as categorias citadas no presente estudo, sendo fundamental como provedor do cuidado, assistência, gestão e acolhimento.

\section{REFERÊNCIAS}

1. BRASIL. Ministério da Saúde (2011). Atenção Humanizada ao Recém-nascido de Baixo Peso: Método Canguru. $2^{2}$ Ed. Brasília: Editora do Ministério da Saúde.

2. CAMPONOGARA S, et al. Percepções Familiares Sobre a Visibilidade do Enfermeiro Atuante em Unidade Intensiva Neonatal e Pediátrica. Revista Contexto \& Saúde. 2018. 18(35); 2176-7114.

3. CARTAXO LS, et al. Vivência de mães na unidade de terapia intensiva neonatal. 2014; 22(4); 551-557.

4. COELHO AS, et al. Nursing team and humanized assistance in neonatal UTI. ReonFacema. 2018; 4(1); 873-877.

5. COSTA R, PADILHA MI. A unidade de Terapia Intensiva Neonatal possibilitando novas práticas no cuidado ao recémnascido. Rev. Gaúcha Enferm. (online). 2011; 32(2); 248-255.

6. COUTO FF, PRAÇA NS. Recém-nascido prematuro suporte materno domiciliar para o cuidado. Ver. Bras. Enferm. 2012. 65(1); 19-26.

7. FERREIRA CM. Unidade de terapia intensiva neonatal: o papel da enfermagem na construção de um ambiente terapêutico. Revista enfermagem do Centro Oeste Mineiro. 2011; 1(2); 268-276.

8. MENDES KDS, et al. Revisão integrativa: método de pesquisa para a Incorporação de Evidências na Saúde e na Enfermagem. Texto Contexo Enferm. 2008; 17(4); 758-764.

9. MENON D, et al. Condições de conforto do paciente internado em UTI neonatal. Caderno da escola de saúde em enfermagem. 2008; 1-15.

10. NASCIMENTO, CAD, et al. Percepção de enfermeiros sobre os pais de prematuros em unidade de terapia Intensiva neonatal. Rene. 2013; 14(4); 811-820.

11. NETO JAS, RODRIGUES BMR. Tecnologias como fundamento do cuidar em neonatologia. Texto contexto Enfermagem. $2010 ; 19(2) ; 372-377$.

12. RODRIGUES LM, MOREIRA PL. Torna-se o pai vivenciando a internação do filho em unidade de Terapia Intensiva Neonatal. J Health Sci Inst. 2012; 30(3); 227-330.

13. SANTANA EFM, MADEIRA KM. A mãe acompanhante na unidade de terapia intensiva de neonatal: desafios para equipe assistencial. Revista de enfermagem do centro de minas gerais. 2013; 3(1); 475-487.

14. SANTOS MSN, et al. Relação familiar na Unidade de Terapia Intensiva Neonatal: Revisão Integrativa. Enferm. foco. 2018. 9 (1): 54-60.

15. SEGANTINI FLC, et al. Cuidado centrado na família em unidade de terapia intensiva neonatal e pediátrica: visão do enfermeiro. Cienc Cuid Saúde. 2018; 17(2); 01-07.

16. SIQUEIRA MBC, DIAS AMB. A percepção materna sobre vivência e aprendizado do cuidado de um bebê prematuro. Epidemiologia e serviço de saúde. 2011. 20(1); 27-36.

17. SOUZA ABG. Recepção e avaliação do recém-nascido. Enfermagem neonatal: cuidado integral ao recém-nascido.2 ed. São Paulo: Atheneu, 2014.

18. STILLWELL SB, et al. Evidence-based practice, step by step: Searching for the evidence. Am J Nurs. 2010; 110(5); 41-7.

19. VERONEZ M, et al. Vivências de mães de bebês prematuros do nascimento a alta: notas de diários de campo. Revista Gaúcha enferm. 2017; 38(2); e60911. 